\title{
Surface Modification of Pigments with Temperature-Responsive Polymer Grafted by Plasma-Induced Polymerization
}

\author{
Tatsuhiko Ihara, Haruo Yukioka, and Yu Iriyama* \\ Department of Industrial Chemistry, Kinki University, \\ 1 Umenobe, Takaya, Higashi-Hiroshima, 739-2116 Japan \\ *Department of Chemistry, Yamanashi University, \\ 4-4-37 Takeda, Kofu, 400-8510 Japan
}

\begin{abstract}
In order to develop temperature-responsive dispersing systems, we have grafted poly(N-isopropylacrylamide) (PNIPA), which is a temperature-responsive polymer having a lower critical solution temperature (LCST) at $32{ }^{\circ} \mathrm{C}$, onto carbon black (CB) by plasmainduced graft polymerization technique. $\mathrm{CB}$ was treated with $\mathrm{H}_{2} \mathrm{O}$ and/or $\mathrm{O}_{2}$ plasma, to which aqueous solution of the monomer (NIPA) was introduced, resulted in simultaneous graft polymerization. The dispersibility of the PNIPA-grafted CB in water at 25 and $35^{\circ} \mathrm{C}$ was investigated by the measurement of the transmittance of visible light $(500 \mathrm{~nm})$. The most of the PNIPA-grafted CB showed better dispersibility at $25^{\circ} \mathrm{C}$ (below the LCST) than at $35^{\circ} \mathrm{C}$ (above the LCST). In many graft polymerization conditions, CB grafted in $2 \mathrm{wt} \%$ of the monomer concentration with ultrasonic irradiation at room temperature showed the most clear temperature response of the dispersibility in water.
\end{abstract}

Keywords: temperature-sensitive polymer, poly(N-isopropylacrylamide), plasmainduced graft polymerization, carbon black, dispersion

\section{Introduction}

Since carbon black (CB) was first found to be grafted with polymers in the 1970 s, many kinds of powders and particles have been reported to be grafted with various polymers as the surface modification. The most of the graftings onto powders were accomplished by the utilization of functional groups on the surface of powders. In the grafting onto $\mathrm{CB}$, since $\mathrm{CB}$ does not have a large amount of functional groups and it is fairly hydrophobic, it is difficult to graft hydrophilic polymers on it.

In our laboratory, we have utilized lowtemperature plasma to graft-polymerize vinyl monomers onto many types of solid surfaces including powders [1-3]. This method, called as plasma-induced graft polymerization, plasma is irradiated to solid surfaces in Stage 1, then liquid monomer solutions are introduced and contacted to the plasma-treated surfaces in Stage 2. Since polymerization-initiating species (radicals) are created in Stage 1, graft polymerization proceeds simultaneously at room temperature in Stage 2. The most fascinating feature of plasma besides the radical formation may be the ability to alter surface property quickly and widely. For example, when hydrophilic plasma gas is used in Stage 1 for CB, the surface of CB becomes hydrophilic. Therefore, hydrophilic monomers can easily start graft polymerization from the CB surface as a good dispersion in a hydrophilic media such as water. When hydrophobic polymer is desired to be grafted, hydrophobic plasma gas ought to be selected. Regardless of the nature of powder surfaces, any kind of vinyl monomer can be graft polymerized by the use of plasma-induced graft polymerization technique.

Poly(N-isopropylacrylamide) (PNIPA) is known as a temperature-responsive or thermosensitive polymer in water [4]. The phase transition of PNIPA occurs at about $32{ }^{\circ} \mathrm{C}$, of which temperature is called lower critical solution temperature, LCST. PNIPA exhibits a sensitive 
and drastic and reversible soluble-insoluble change in water at below and above the LCST: At temperatures below the LCST, PNIPA is surrounded with water and hydrated, which makesclear aqueous solution, while at temperatures above the LCST, dehydration occurs and hydrophobic interaction increases, which makes the solution clouded. Because of this unique thermal property, PNIPA has been applied widely. For example, PNIPAcontaining gel has been studied extensively for a new drug delivery system [5]. Immobilization of enzymes by PNIPA is another attractive field because its reactivity and solubility can be controlled easily by a slight change of temperature [6-8]. Microsphere of PNIPA has been also prepared [9].

Grafting of PNIPA on solid surfaces also opens more application fields [10]. There are quite a few reports on the application of PNIPA grafting on packing materials of chromatographies. By the grafting of PNIPA on packing materials, elution pattern changes dramatically at different temperatures [11-13].

There are some methods in grafting. Plasma is one of the easiest methods to graft polymers onto solid surfaces, and many researchers have been grafted many kinds of vinyl monomers onto various solid materials. Lee et al. grafted PNIPA onto porous polyamide membrane and used for a selective permeation [14].

In this study, we grafted PNIPA onto CB surface by plasma-induced graft polymerization and investigated the temperature dependence of the dispersibility of PNIPA-grafted CB in water.

\section{Experimental}

2.1. Materials

CB used in this study was high abrasion furnace $\mathrm{CB}$ for a rubber-reinforcement use. The specific surface area of the CB was $68 \mathrm{~m}^{2} / \mathrm{g}$. As a grafting monomer, NIPA, purchased from Wako Chemical Co., was used after recrystallization. Water used in this study, as a solvent of NIPA, plasma gas, water-absorption test, and dispersion medium, was distilled and ion-exchanged before use. Water as a plasma gas and the monomer solution were put in ampules and degassed by a ultrasonic irradiation under vacuum before the plasma treatment, and connected to the reactor.

\subsection{Plasma-Induced Graft Polymerization}

The plasma reactor system used in this study is shown in Figure 1. This reactor system wasspecially designed for plasma-induced graft polymerization for powdery materials, which equipped with a rotatable round-bottomed flask and plasma gas inlets and a liquid monomer inlet. The rotatable reactor enables uniform plasma treatment of powdery materials.

The plasma-induced graft polymerization of NIPA onto CB was carried out in the following manner.

Stage 1: CB (1 g) was put into the roundbottomed flask. The reactor system was evacuated by a rotary vacuum pump to the pressure of lower than $0.1 \mathrm{~Pa}$. Then $\mathrm{H}_{2} \mathrm{O}$ and/or $\mathrm{O}_{2}$ was supplied to the reactor through a needle valve for the system pressure to be $67 \mathrm{~Pa}$. When $\mathrm{H}_{2} \mathrm{O}$ and $\mathrm{O}_{2}$ was

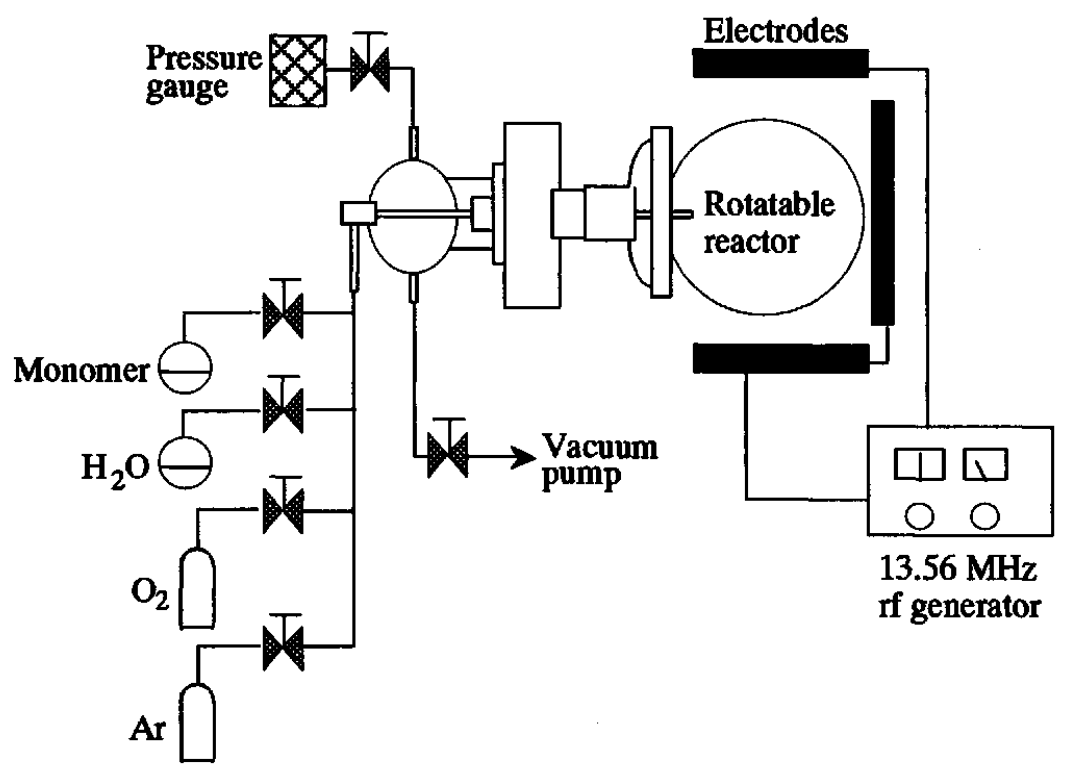

Figure 1. Schematic illustration of plasma reactor system for plasma-induced graft polymerization for powdery materials. 
mixed, the mixing ratio was $1: 1$, in which water was introduced first to be $33 \mathrm{~Pa}$, then $\mathrm{O}_{2}$ was added to be the total pressure of $67 \mathrm{~Pa}$. Plasma was then generated in the reactor flask, which was surrounded by electrodes connected to a $13.56-\mathrm{MHz}$ if power supply. The plasma treatment of $\mathrm{CB}$ was done for $10 \mathrm{~min}$. During the plasma treatment, the flask was kept rotating at about $10 \mathrm{rpm}$ for the uniform treatment.

Stage 2: $30 \mathrm{~mL}$ of aqueous solution of NIPA (1-3 wt\%), which was previously degassed, was introduced to the flask immediately after the plasma treatment. In this process, the monomer solution was easily sucked up and replaced to the reactor flask because the pressure of the system inside was much lower. After the monomer solution was replaced completely, the system pressure was raised to atmospheric pressure with Ar gas in order to prevent air (oxygen) to come in. Graft polymerization takes place simultaneously when CB surface contacts with the monomer since initiating species(radical) have been already created by the plasma treatment. Therefore, initiator or heating process is not necessary in plasma-induced graft polymerization. The graft polymerization was carried out at room temperature for $24 \mathrm{~h}$ unless otherwise specified. For the uniform grafting on $\mathrm{CB}$, the reactor was irradiated by ultrasonic during the graft polymerization.

The products were washed with water for the removal of monomer and homopolymer. PNIPAgrafted CB was obtained after centrifugal separation, followed by vacuum drying.

\subsection{Properties of PNIPA-Grafted CB}

\subsubsection{Water-Absorption Test}

Water-absorption test was carried out for the evaluation of hydrophilicity of plasma-treated CB. $0.3 \mathrm{~g}$ of untreated and plasma-treated $\mathrm{CB}$ powders were reshaped to be a pellet (10-mm d.) by pressing at $290 \mathrm{MPa}$ for $5 \mathrm{~min}$. Ten microliters of water was placed on the $C B$ pellet. Time the $C B$ pellet required to absorb the water drop completely was measured by the captured image with a digital video camera (Sony DCR-VX 1000).

\subsubsection{Thermal Analysis}

Differential scanning calorimetry (DSC) was measured with a differential scanning calorimeter (Seiko Denshi DSC220C) for PNIPA polymerized with an initiator, $\mathrm{H}_{2} \mathrm{O}_{2}$. The temperature was increased at a rate of $2{ }^{\circ} \mathrm{C} / \mathrm{min}$. The differential curve of DSC (DDSC) was also recorded.

2.3.3. Dispersibility and Its Temperature Response of $\mathrm{CB}$ in Water
Dispersibility of CB in water was evaluated by the measurement of the transmittance of its dispersion. In this experiment, we used water of pH 2 (prepared with $\mathrm{HCl}$ ), in order to prevent the dissociation of acidic groups formed on the surface by the plasma treatment, which can keep good dispersibility even at higher temperature than the LCST. The dispersion was prepared in a sample bottle with $10 \mathrm{~mL}$ of water and $10 \mathrm{mg}$ of $\mathrm{CB}$ by the irradiation of ultrasonic for $10 \mathrm{~min}$. The dispersion was replaced into an optical cell and left still in an incubator at 25 or $35{ }^{\circ} \mathrm{C}$ (or given temperature) for $4 \mathrm{~h}$, then transmittance of light $(500 \mathrm{~nm})$ was measured with a UV-vis absorption spectrometer (Shimadzu UV-1200).

In order to examine the temperature response of the CB dispersibility, we successively measured transmittance of CB dispersion in the following manner. First, CB dispersion in water was prepared at $35^{\circ} \mathrm{C}$ in the same way as stated above, and transmittance was measured after $4 \mathrm{~h}$. Then, the dispersion was irradiated again with ultrasonic at $25^{\circ} \mathrm{C}$, and transmittance was measured after $4 \mathrm{~h}$. This ultrasonic dispersion-measurement at $25{ }^{\circ} \mathrm{C}$ was performed three times consecutively. We define the above four measurements, one at $35{ }^{\circ} \mathrm{C}$ and three at $25^{\circ} \mathrm{C}$, ONE sequence. After the first sequence, temperature was raised to $35^{\circ} \mathrm{C}$ and the second sequence was started. For each sample, we repeated three sequences and finished with the measurement at $35^{\circ} \mathrm{C}$, altogether 13 measurements. In the figures exhibiting transmittance, the lower the transmittance is, the greater the dispersibility

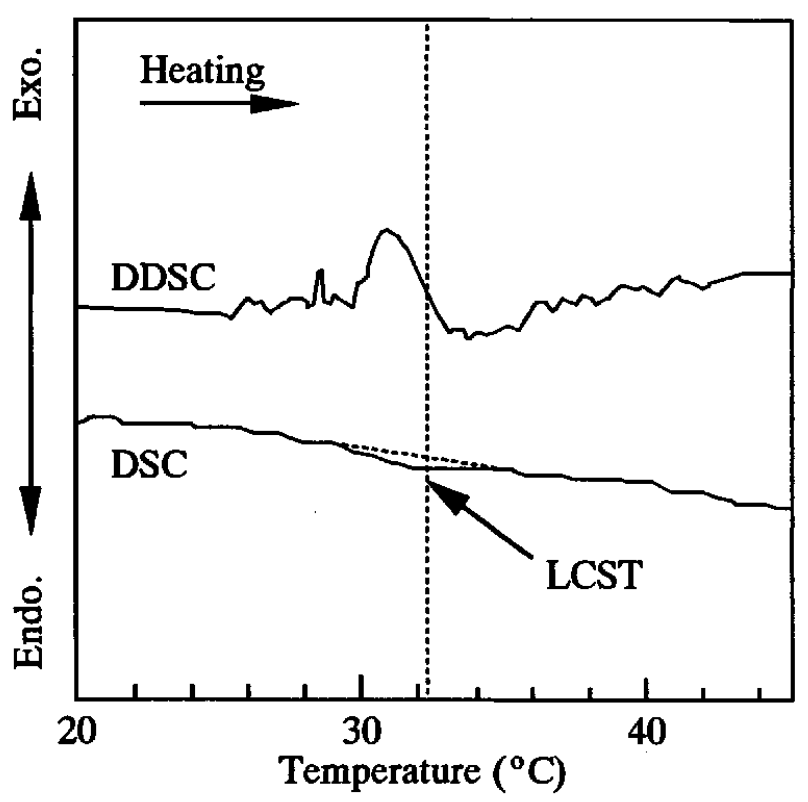

Figure 2. DSC and DDSC curves of PNIPA. 
Table 1. Time required for $\mathrm{CB}$ pellets to absorb a $10-\mu \mathrm{L}$ water droplet.

\begin{tabular}{cc}
\hline Treatment of CB & Time (s) \\
\hline None & 7920 \\
$\mathrm{H}_{2} \mathrm{O}$ plasma & 4.0 \\
$\mathrm{H}_{2} \mathrm{O}-\mathrm{O}_{2}$ plasma & 2.7 \\
$\mathrm{O}_{2}$ plasma & 0.1 \\
\hline
\end{tabular}

is, because when CB disperses well, the whole dispersion looks dark, and transmittance becomes almost zero.

\section{Results and Discussion}

\subsection{Thermal Analysis of PNIPA}

DSC and DDSC of PNIPA, polymerized in an aqueous solution with $\mathrm{H}_{2} \mathrm{O}_{2}$ as an initiator, in the temperature range between 20 and $45{ }^{\circ} \mathrm{C}$ with temperature-increasing rate of $2{ }^{\circ} \mathrm{C} / \mathrm{min}$ are shown in Figure 2. In DSC curve, a small and broad endothermic peak can be found at about $32{ }^{\circ} \mathrm{C}$ resulted from the phase transfer of PNIPA, as many literatures have described. From a closer analysis of the DDSC curve, the endothermic phase transfer of PNIPA was found to start at $29.3^{\circ} \mathrm{C}$ and end at $37.0{ }^{\circ} \mathrm{C}$ and to have the endothermic peak, LCST, at $32.3^{\circ} \mathrm{C}$. The enthalpy, $\Delta \mathrm{H}$, of the phase transfer obtained from the endothermic peak area of the DSC curve was $15 \mathrm{~J} / \mathrm{g}$. In general, $\Delta \mathrm{H}$ of phase transfer for organic polymers is small, but that of PNIPA can be said one of the smallest.

\subsection{Property of Plasma-Treated CB Surface}

After the plasma treatment with $\mathrm{H}_{2} \mathrm{O}$ and/or $\mathrm{O}_{2}$, the surface of $\mathrm{CB}$ became hydrophilic because those plasma gases are hydrophilic or effective to create hydrophilic groups. For the evaluation of hydrophilicity, the absorbability of water was measured. Table 1 shows the time that CB pellet required to absorb a $10-\mathrm{mL}$ water drop. Without plasma treatment, it took more than two hours. With plasma treatments with $\mathrm{H}_{2} \mathrm{O}, \mathrm{H}_{2} \mathrm{O}-\mathrm{O}_{2}$, and $\mathrm{O}_{2}$, the time dramatically shortened to $4.0,0.3$, and $0.1 \mathrm{~s}$, respectively. It was very effective for those plasma to alter the CB surface to hydrophilic. In addition to radical formation, this hydrophilic surface modification enables effective and uniform graft polymerization of aqueous monomers. In the comparison between $\mathrm{H}_{2} \mathrm{O}$ and $\mathrm{O}_{2}$ plasmas, $\mathrm{O}_{2}$ plasma was more effective in hydrophilic modification probably because hydrophilic groups created were effective.

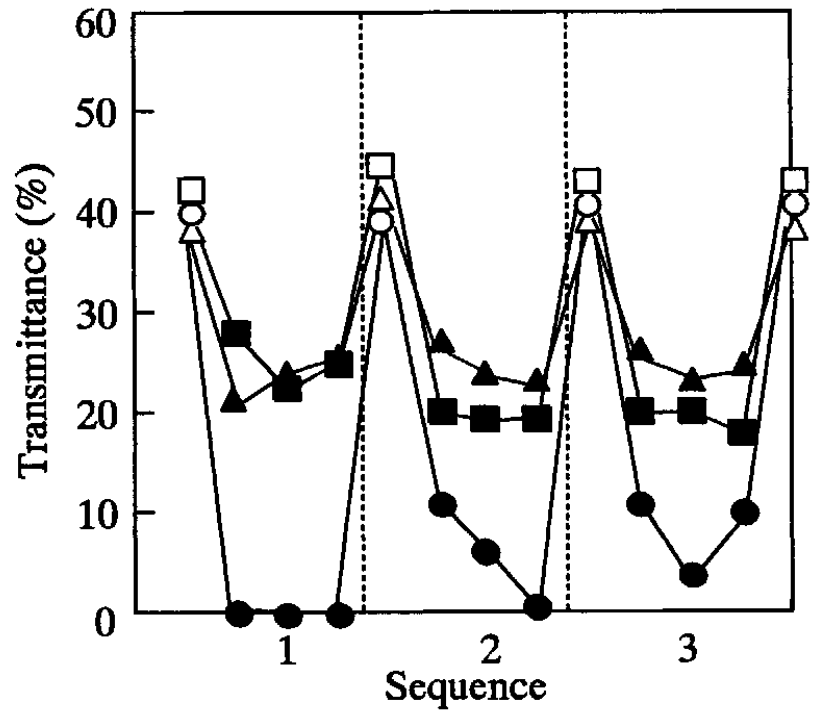

Figure 3. Transmittance of the dispersion of PNIPAgrafted $\mathrm{CB}$ in water for three consecutive sequences of temperature change.

Open symbols, $35^{\circ} \mathrm{C}$; closed symbols, $25^{\circ} \mathrm{C}$.

$\triangle \Delta$, Grafted without ultrasonic irradiation at room temperature; $O O$, with ultrasonic irradiation at room temperature; $\square$, with ultrasonic irradiation at $60^{\circ} \mathrm{C}$. Plasma, $\mathrm{H}_{2} \mathrm{O}-\mathrm{O}_{2}$; monomer concn, $2 \mathrm{wt} \%$.

\subsection{Dispersibility of PNIPA-Grafted CB}

In order to evaluate of the dispersibility of CB, we measured the transmittance of light $(500 \mathrm{~nm})$ passed through an optical cell containing dispersion of $\mathrm{CB}$ in water. Figure 3 shows transmittance of the dispersion of PNIPA-grafted CB in water for three consecutive sequences of temperature change as stated in the experimental section: Each sequence consists of a measurement at $35^{\circ} \mathrm{C}$, followed by three measurements at $25^{\circ} \mathrm{C}$. In this figure, three kinds of $\mathrm{CB}$ are compared. All three $\mathrm{CB}$ were treated with $\mathrm{H}_{2} \mathrm{O}-\mathrm{O}_{2}$ mixture plasma in Stage 1, but the condition of graft polymerization in Stage 2 was different as follows: (1) without ultrasonic irradiation at room temperature; (2) with ultrasonic irradiation at room temperature; (3) with ultrasonic irradiation at $60^{\circ} \mathrm{C}$. At the beginning of the first sequence, all three samples at $35^{\circ} \mathrm{C}$ (open symbols) showed transmittance about $40 \%$, which implies poor dispersibility. Next, when the temperature was lowered to $25{ }^{\circ} \mathrm{C}$ (closed symbols), transmittance for all three decreased. Among them, that of CB grafted with ultrasonic at room temperature showed the lowest transmittance, zero, which indicates excellent dispersibility. In the comparison between 25 and $35^{\circ} \mathrm{C}$, PNIPA-grafted $\mathrm{CB}$ showed better dispersibility at $25{ }^{\circ} \mathrm{C}$ than 35 ${ }^{\circ} \mathrm{C}$. The reason is that, as we stated repeatedly, PNIPA has an LCST at $32^{\circ} \mathrm{C}$ : At above the LCST, grafted PNIPA on CB shrinks because of 
hydrophobic interaction, which results in poor dispersibility.

In the 2nd sequence in Fig. 3, when the temperature was raised to $35{ }^{\circ} \mathrm{C}$ again, the transmittance for all CB showed almost the same transmittance as at the beginning of the 1st sequence. When temperature was lowered to $25^{\circ} \mathrm{C}$ again and measured three times in the 2nd sequence, although the transmittance for CB grafted with ultrasonic at room temperature, which is the lowest among the three, did not fell to as low as in the 1st sequence, the pattern of the transmittance for all CB was very similar to the 1st sequence.

The 3 rd sequence was also very much similar to the 2nd sequence. The similarity of the transmittance pattern, or dispersibility change, proved the reversible temperature response in the phase transfer of grafted PNIPA on CB. Without ultrasonic, probably NIPA did not grafted on CB uniformly and there were many $\mathrm{CB}$ particles which had not been grafted. With ultrasonic at $60{ }^{\circ} \mathrm{C}$, NIPA may have been grafted uniformly, but since the temperature was above the LCST, CB particles might have aggregated because of the hydrophobic interaction of PNIPA and aggregated gradually during the polymerization process.

Next, we compared the effect of the monomer concentration in the graft polymerization on the dispersibility. Figure 4, looks similar to Fig. 3, shows transmittance of the dispersion of PNIPAgrafted CB for three sequences of the temperature

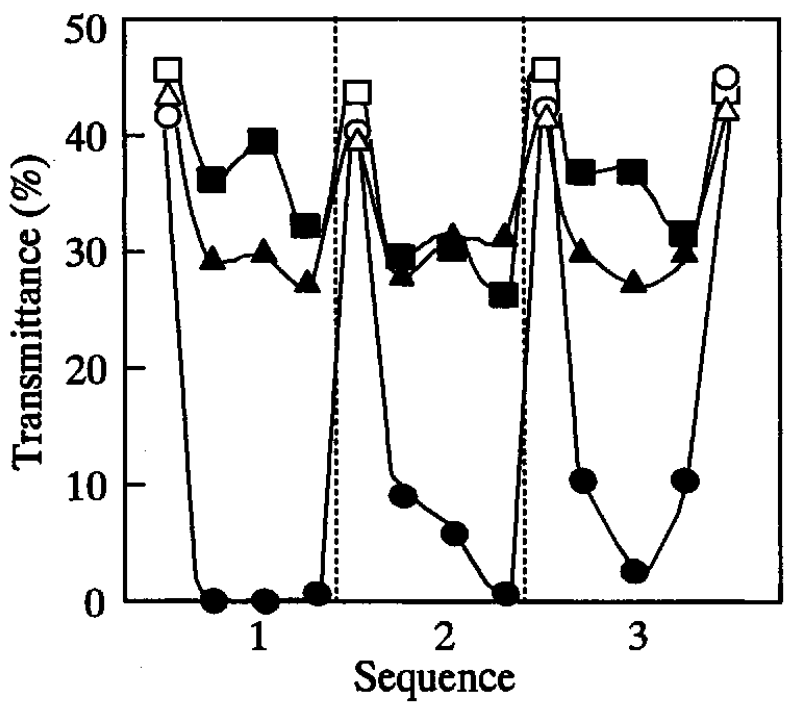

Figure 4. Transmittance of the dispersion of PNIPAgrafted CB in water for three consecutive sequences of temperature change.

Open symbols, $35^{\circ} \mathrm{C}$; closed symbols, $25^{\circ} \mathrm{C}$.

Grafted with ultrasonic irradiation at room temperature in monomer concentration of $3 \mathrm{wt} \%(\Delta \triangle), 2$

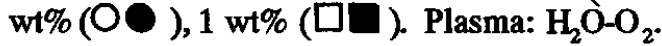

change for $\mathrm{CB}$ grafted with different concentrations of NIPA with ultrasonic irradiation at room temperature. Plasma treatment gas in Stage 1 was $\mathrm{H}_{2} \mathrm{O}-\mathrm{O}_{2}$ mixture. In this figure, $\mathrm{CB}$ grafted in NIPA concentration of $2 \mathrm{wt} \%$ showed the most clear reversible temperature response, while those in 1 and $3 \mathrm{wt} \%$ did not achieve good dispersibility. CB grafted in 1 wt\% NIPA seems to have too little grafted polymer on to maintain good dispersion. That grafted in $3 \mathrm{wt} \%$ seems to have been aggregated during the graft polymerization because of a little too long or dense polymer chains.

Figure 5 shows transmittance of the dispersion of PNIPA-grafted CB at various temperatures from 20 to $40{ }^{\circ} \mathrm{C}$ along with the DSC curve of PNIPA (same as Fig. 2). The all measurements were done $4 \mathrm{~h}$ after the ultrasonic dispersion. The $\mathrm{CB}$ used in this figure was plasma-treated with $\mathrm{H}_{2} \mathrm{O}-\mathrm{O}_{2}$ and grafted in $2 \mathrm{wt} \%$ of NIPA with ultrasonic at room temperature. The transmittance was almost zero, or dispersibility was excellent, at 20 and $25^{\circ} \mathrm{C}$, but it started increasing at $28{ }^{\circ} \mathrm{C}$, then it reached the maximum at $34^{\circ} \mathrm{C}$ and was constant after that. In the DSC curve, the endothermic peak can be observed from 29 to $35^{\circ} \mathrm{C}$, which indicate the phase change of grafted PNIPA. The temperatures for the two property changes coincided each other.

Figure 6 shows the transmittance of the dispersion of PNIPA-grafted CBfor three sequences in the comparison between $\mathrm{H}_{2} \mathrm{O}-\mathrm{O}_{2}$ and $\mathrm{O}_{2}$ plasmas. Both the CB were grafted in $2 \mathrm{wt} \%$ of NIPA with

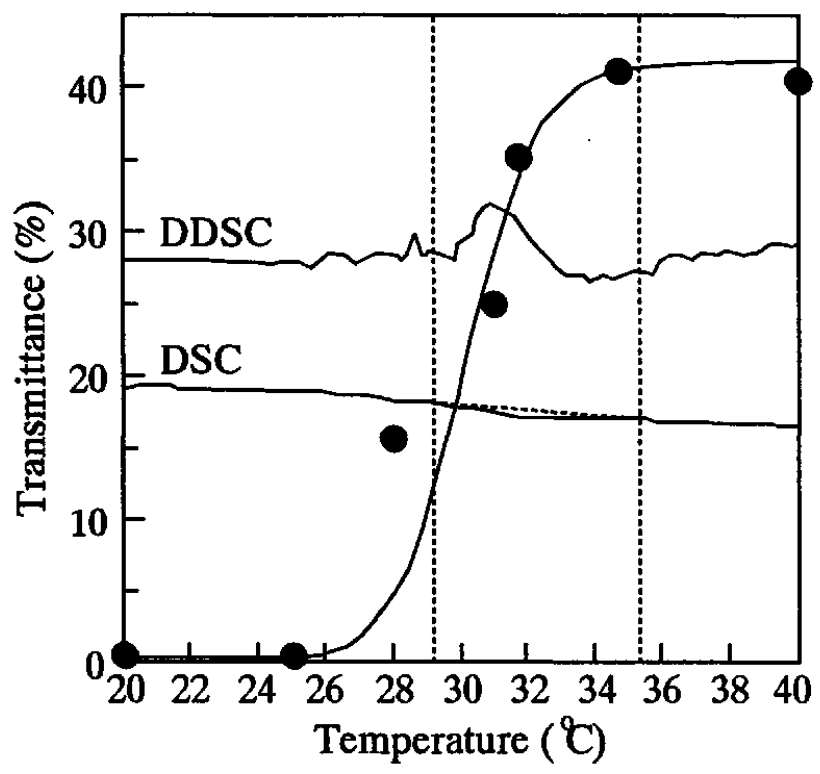

Figure 5. Transmittance of the dispersion of PNIPAgrafted $\mathrm{CB}$ at temperatures between 20 and $40^{\circ} \mathrm{C}$ and its comparison with DSC and DDSC curves.

Grafted in monomer concn. of $2 \mathrm{wt} \%$ with ultrasonic irradiation at room temperature. Plasma: $\mathrm{H}_{2} \mathrm{O}-\mathrm{O}_{2}$. 


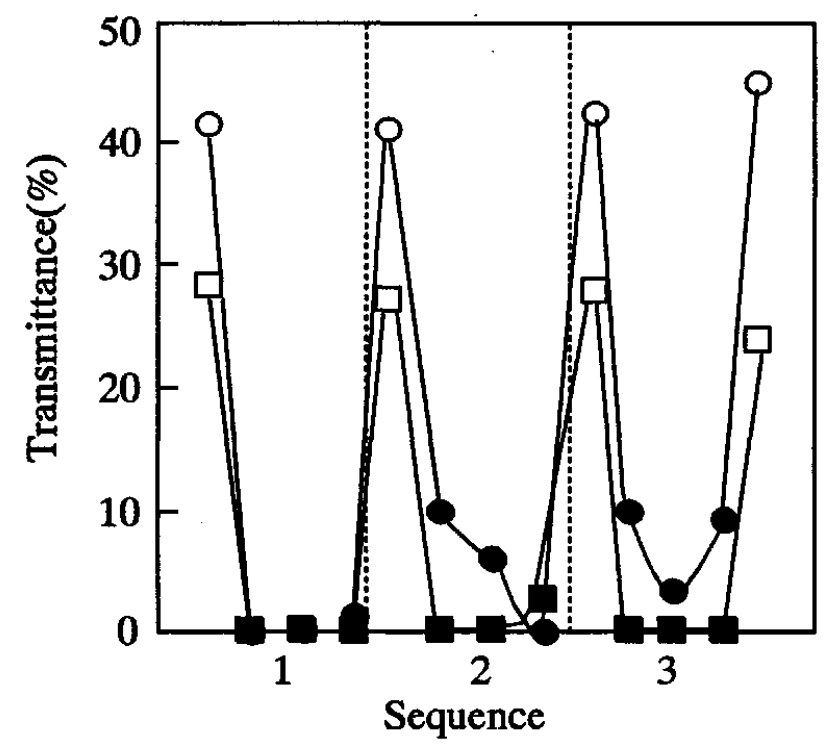

Figure 6. Transmittance of the dispersion of PNIPAgrafted CB in water for three consecutive sequences of temperature change.

Open symbols, $35^{\circ} \mathrm{C}$; closed symbols, $25^{\circ} \mathrm{C}$ $\mathrm{OO}, \mathrm{H}_{2} \mathrm{O}-\mathrm{O}_{2}$ plasma treated in Stage $1 ; \square \square, \mathrm{O}_{2}$ plasma treated in Stage 1.

Grafted in monomer concentration of $2 \mathrm{wt} \%$ with ultrasonic irradiation at room temperature.

ultrasonic at room temperature. $\mathrm{O}_{2}$ plasma-treated $\mathrm{CB}$, similar to $\mathrm{H}_{2} \mathrm{O}-\mathrm{O}_{2}$ treated one, showed temperature dependence on the transmittance: It dispersed poorly at $35^{\circ} \mathrm{C}$ while it dispersed excellent at $25^{\circ} \mathrm{C}$. The transmittance of $\mathrm{O}_{2}$ treated $\mathrm{CB}$ was lower than that of $\mathrm{H}_{2} \mathrm{O}-\mathrm{O}_{2}$ treated one at $35{ }^{\circ} \mathrm{C}$, and those of all $\mathrm{O}_{2}$ treated one at $25{ }^{\circ} \mathrm{C}$ were zero. We can say the transmittance for $\mathrm{O}_{2}$ treated $\mathrm{CB}$ shifted a little lower than that for $\mathrm{H}_{2} \mathrm{O}-\mathrm{O}_{2}$ treated one. The reason for this difference probably comes from the difference in the hydrophilicity of CB just after the plasma treatment described in Table 1. That is, $\mathrm{O}_{2}$ treated $\mathrm{CB}$ was more hydrophilic than that treated with $\mathrm{H}_{2} \mathrm{O}-\mathrm{O}_{2}$ plasma. Therefore, even after the graft polymerization, the surface of $\mathrm{O}_{2}$ treated $\mathrm{CB}$ may be relatively hydrophilic. We donot have complete results for $\mathrm{H}_{2} \mathrm{O}$ plasma-treated CB correspond to Fig. 6, but its dispersibility at 25 ${ }^{\circ} \mathrm{C}$ was not as good as the others, as expected from the results in Table 1.

\section{Conclusion}

PNIPA was grafted onto CB by plasma-induced graft polymerization. In the plasma treatment of CB in Stage 1, $\mathrm{H}_{2} \mathrm{O}$ and/or $\mathrm{O}_{2}$ plasma was used, which altered the CB surface drastically to hydrophilic in addition to the radical formation. Therefore, aqueous solution of NIPA was easy to contact with CB in Stage 2 and graft polymerization proceeded uniformly in water. The most PNIPAgrafted CB showed temperature dependence of its dispersibility in water, evaluated by the transmittance of light: The dispersibility was better at $25^{\circ} \mathrm{C}$ (below the LCST of PNIPA) than at $35^{\circ} \mathrm{C}$ (above the LCST). The most clear temperature response was observed in the PNIPA-grafted CB polymerized in 2 wt\% NIPA with ultrasonic irradiation at room temperature. Between plasma treatments with $\mathrm{H}_{2} \mathrm{O}-\mathrm{O}_{2}$ and $\mathrm{O}_{2}$, PNIPA-grafted CB treated with the latter in Stage 1 showed a little better dispersibility at both 25 and $35^{\circ} \mathrm{C}$.

\section{Acknowledgments}

This work was partly supported by Foundation for Promotion of Material Science and Technology of Japan (MST Foundation).

\section{References}

1. Y. Osada and Y. Iriyama, Thin Solid Films, 118 (1984) 197.

2. Y. Iriyama and S. Ikeda, Polym. J., 26 (1994) 109.

3. T. Ihara, N. Asazaki, and M. Kiboku, Shikizai Kyokaishi, 69 (1996) 307.

4. M. Heskins and J. E. Guillet, J. Macromol. Sci.-Chem., A2 (1968) 1441.

5. Y. Kaneko, R. Yoshida, K. Sakai, Y. Sakurai, and T. Okano, J. Membr. Sci., 101 (1995) 13.

6. K. Hoshino, M. Katagiri, M. Taniguchi, T. Sasakura, and M. Fujii, J. Ferment. Bioeng., 77 (1994) 407.

7. M. Matsukata, Y. Takei, T. Aoki, K. Sanui, N. Ogata, Y. Sakurai, and T. Okano, J. Biochem., 116 (1994) 682.

8. H. Nagayama, Y. Maeda, C. Shimasaki, and H. Kitano, Macromol. Chem. Phys., $196(1995) 611$.

9. H. Kitano and J. Kawabata, Macromol. Chem. Phys., 197 (1996) 1721.

10. Y. G. Takei, T. Aoki, K. Sanui, N. Ogata, Y. Sakurai, and T. Okano, Macromolecules, 27 (1994) 6163.

11. K. Hosoya, K. Kimata, T. Araki, N. Tanaka, and J. M. J. Frechet, Anal. Chem., 67 (1995) 1907. 12. H. Kanazawa, K. Yamamoto, Y. Matsushima, N. Takai, A. Kikuchi, T. Okano, and Y. Sakurai, Anal. Chem., 68 (1996) 100.

13. H. Kanazawa, K. Yamamoto, Y. Kashiwase, Y. Matsushima, N. Takai, A. Kikuchi, Y. Sakurai, and T. Okano, J. Pharm. Biomed. Anal., 15 (1997) 1545.

14. Y. M. Lee and J. K. Shim, Polymer, 38 (1997) 1227. 